\title{
VGEA: An RNA viral assembly toolkit
}

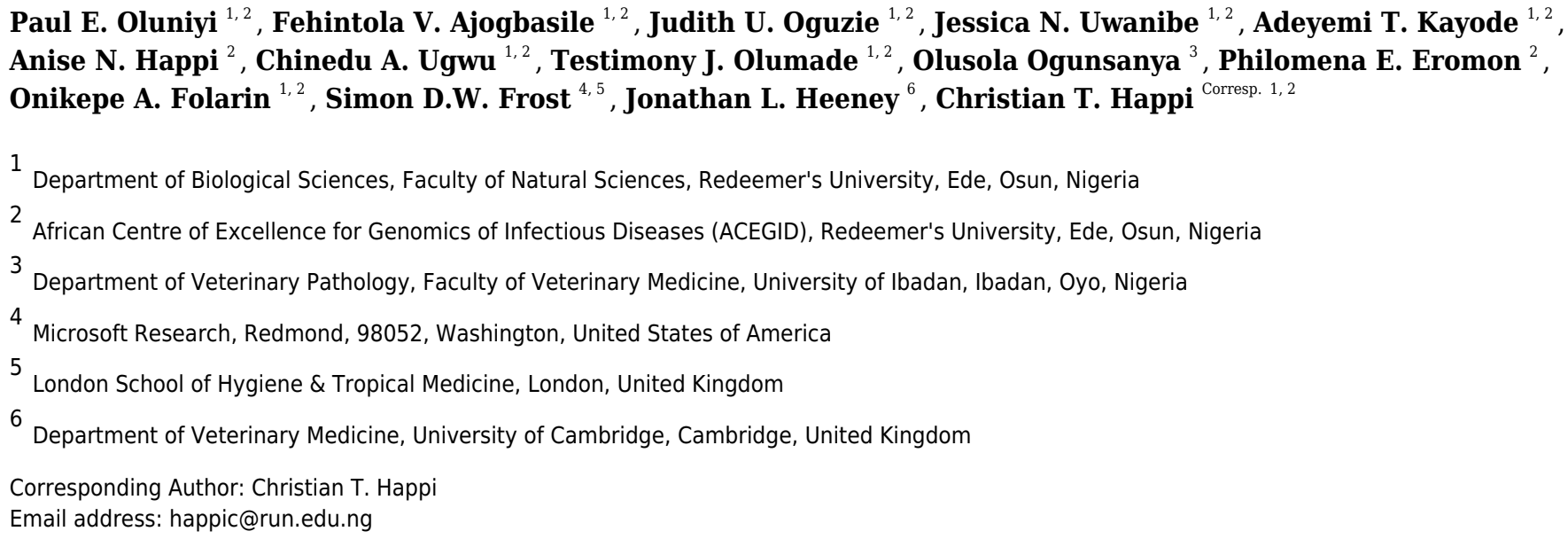

Next generation sequencing (NGS)-based studies have vastly increased our understanding of viral diversity. Viral sequence data obtained from NGS experiments are a rich source of information, these data can be used to study their epidemiology, evolution, transmission patterns, and can also inform drug and vaccine design. Viral genomes however represent a great challenge to bioinformatics due to their high mutation rate and forming quasispecies in the same infected host, bringing about the need to implement advanced bioinformatics tools to assemble consensus genomes well-representative of the viral population circulating in individual patients. Many tools have been developed to preprocess sequencing reads, carry-out de novo or reference-assisted assembly of viral genomes and assess the quality of the genomes obtained. Most of these tools however exist as standalone workflows and usually require huge computational resources. Here we present VGEA (Viral Genomes Easily Analyzed), a Snakemake workflow for analyzing RNA viral genomes. VGEA enables users to map sequencing reads to the human genome to remove human contaminants, split bam files into forward and reverse reads, carry out de novo assembly of forward and reverse reads to generate contigs, pre-process reads for quality and contamination, map reads to a reference tailored to the sample using corrected contigs supplemented by the user's choice of reference sequences and evaluate/compare genome assemblies. We designed a project with the aim of creating a flexible, easy-to-use and all-in-one pipeline from existing/stand-alone bioinformatics tools for viral genome analysis that can be deployed on a personal computer. VGEA was built on the Snakemake workflow management system and utilizes existing tools for each step: fastp (Chen et al., 2018) for read trimming and read-level quality control, BWA ( $L i$ and Durbin, 2009) for 
mapping sequencing reads to the human reference genome, SAMtools (Li et al., 2009) for extracting unmapped reads and also for splitting bam files into fastq files, IVA (Hunt et al., 2015) for de novo assembly to generate contigs, shiver (Wymant et al., 2018) to preprocess reads for quality and contamination, then map to a reference tailored to the sample using corrected contigs supplemented with the user's choice of existing reference sequences, SeqKit (Shen et al., 2016) for cleaning shiver assembly for QUAST, QUAST (Gurevich et al., 2013) to evaluate/assess the quality of genome assemblies and MultiQC (Ewels et al., 2016) for aggregation of the results from fastp, BWA and QUAST. Our pipeline was successfully tested and validated with SARS-CoV-2 $(n=20)$, HIV-1 $(n=20)$ and Lassa Virus $(n=20)$ datasets all of which have been made publicly available. VGEA is freely available on GitHub at: https://github.com/pauloluniyi/VGEA under the GNU General Public License. 


\section{VGEA: An RNA viral assembly toolkit}

2

3 Paul E. Oluniyi ${ }^{1,2}$, Fehintola V. Ajogbasile ${ }^{1,2}$, Judith U. Oguzie ${ }^{1,2}$, Jessica N. Uwanibe ${ }^{1,2}$,

4 Adeyemi T. Kayode ${ }^{1,2}$, Anise N. Happi ${ }^{2}$, Chinedu A. Ugwu ${ }^{1,2}$, Testimony J. Olumade ${ }^{1,2}$, Olusola

5 Ogunsanya $^{3}$, Philomena E. Eromon ${ }^{2}$, Onikepe A. Folarin ${ }^{1,2}$, Simon D.W. Frost ${ }^{4,5}$, Jonathan L.

6 Heeney $^{6}$, Christian T. Happi ${ }^{1,2^{*}}$

7

8 1. Department of Biological Sciences, Faculty of Natural Sciences, Redeemer's University, Ede, 9 Osun State, Nigeria.

10 2. African Centre of Excellence for Genomics of Infectious Diseases (ACEGID), Redeemer's

11 University, Ede, Osun State, Nigeria.

12 3. Department of Veterinary Pathology, Faculty of Veterinary Medicine, University of Ibadan,

13 Ibadan, Nigeria.

14 4. Microsoft Research, Redmond, 98052, WA, USA.

15 5. London School of Hygiene \& Tropical Medicine, London, UK.

16 6. Department of Veterinary Medicine, University of Cambridge, Cambridge, United Kingdom.

19 *Corresponding Author:

20 Christian Happi ${ }^{1,2}$

21 Email address: happic@,run.edu.ng 


\section{ABSTRACT}

25 Next generation sequencing (NGS)-based studies have vastly increased our understanding of

26 viral diversity. Viral sequence data obtained from NGS experiments are a rich source of

27 information, these data can be used to study their epidemiology, evolution, transmission patterns,

28 and can also inform drug and vaccine design. Viral genomes however represent a great challenge

29 to bioinformatics due to their high mutation rate and forming quasispecies in the same infected

30 host, bringing about the need to implement advanced bioinformatics tools to assemble consensus

31 genomes well-representative of the viral population circulating in individual patients. Many tools

32 have been developed to preprocess sequencing reads, carry-out de novo or reference-assisted

33 assembly of viral genomes and assess the quality of the genomes obtained. Most of these tools

34 however exist as standalone workflows and usually require huge computational resources. Here

35 we present VGEA (Viral Genomes Easily Analyzed), a Snakemake workflow for analyzing

36 RNA viral genomes. VGEA enables users to map sequencing reads to the human genome to

37 remove human contaminants, split bam files into forward and reverse reads, carry out de novo

38 assembly of forward and reverse reads to generate contigs, pre-process reads for quality and

39 contamination, map reads to a reference tailored to the sample using corrected contigs

40 supplemented by the user's choice of reference sequences and evaluate/compare genome

41 assemblies. We designed a project with the aim of creating a flexible, easy-to-use and all-in-one

42 pipeline from existing/stand-alone bioinformatics tools for viral genome analysis that can be

43 deployed on a personal computer. VGEA was built on the Snakemake workflow management

44 system and utilizes existing tools for each step: fastp (Chen et al., 2018) for read trimming and

45 read-level quality control, BWA (Li and Durbin, 2009) for mapping sequencing reads to the

46 human reference genome, SAMtools (Li et al., 2009) for extracting unmapped reads and also for 
47 splitting bam files into fastq files, IVA (Hunt et al., 2015) for de novo assembly to generate 48 contigs, shiver (Wymant et al., 2018) to pre-process reads for quality and contamination, then 49 map to a reference tailored to the sample using corrected contigs supplemented with the user's 50 choice of existing reference sequences, SeqKit (Shen et al., 2016) for cleaning shiver assembly

51 for QUAST, QUAST (Gurevich et al., 2013) to evaluate/assess the quality of genome

52 assemblies and MultiQC (Ewels et al., 2016) for aggregation of the results from fastp, BWA and 53 QUAST. Our pipeline was successfully tested and validated with SARS-CoV-2 (n = 20), HIV-1

$54(\mathrm{n}=20)$ and Lassa Virus $(\mathrm{n}=20)$ datasets all of which have been made publicly available.

55 VGEA is freely available on GitHub at: https://github.com/pauloluniyi/VGEA under the GNU 56 General Public License.

INTRODUCTION

The most abundant biological entities on Earth are viruses as they can be found among all cellular forms of life. So far, over four thousand five hundred viral species have been discovered, from which a huge amount of sequence information has been collected by researchers and scientists all over the world (Pickett et al., 2012; Sharma et al., 2015; Brister et al., 2015). In recent times (past two decades), a number of these viruses have emerged in the human population causing disease outbreaks and sometimes pandemics. These viruses include mainly: Influenza virus, Severe Acute Respiratory Syndrome (SARS) coronavirus, Middle East Respiratory Syndrome (MERS) coronavirus, Ebola virus, Yellow fever virus, Lassa virus (LASV), Zika virus (Chan, 2002; Bean et al., 2013; Folarin et al., 2016; Grubaugh et al., 2017; Metsky et al., 2017; Siddle et al., 2018; Ajogbasile et al., 2020) and SARS-CoV-2 (Chen et al., 2020; Holshue et al., 2020; Sohrabi et al., 2020). During these outbreaks and pandemics, 
70 genomic sequencing for identification and characterization of the transmission and evolution of

71 the causative agents have proved to be critical in helping inform disease surveillance and

72 epidemiology.

73 Next Generation Sequencing (NGS) platforms have been widely accepted as high-throughput,

74 open view technologies that have many attractive features for virus detection and assembly

75 (Tang \& Chiu, 2010; Mokili et al., 2012). NGS-based studies have vastly increased our

76 understanding of viral diversity (Reyes et al., 2010; Cantalupo et al., 2011). Pathogen sequence

77 data obtained from NGS experiments are a rich source of information, these data can be used to

78 study their epidemiology, evolution, transmission patterns, and can also inform drug and vaccine

79 design. The field of genomics, especially pathogen genomics has been transformed by NGS, with

80 costs constantly decreasing, equipment becoming more portable/field deployable during

81 outbreaks and remarkable increase in data availability.

82 The huge amount of data being generated requires various processing steps such as removal of

83 primers and adapters, quality filtering and control which is usually crucial for various

84 downstream analysis. Several tools have been developed for these purposes, such as fastp (Chen

85 et al., 2018) and Trimmomatic (Bolger et al., 2014).

86 Reconstructing viral genomes from NGS data is usually achieved through de novo assembly

87 (which is the process of assembling genomes using overlapping sequencing reads), or through a

88 reference-guided approach (which involves mapping sequence reads to a reference genome).

89 Numerous tools have been developed for these purposes; SPAdes (Bankevich et al., 2012),

90 Burrows-Wheeler Alignment tool (BWA), V-GAP (Nakamura, 2016), VirusTAP (Yamashita,

91 2016), V-Pipe (Posada-Céspedes, 2021) and viral-ngs (https://github.com/broadinstitute/viral- 
92 ngs), amongst others. Contigs generated by de novo assembly however do not provide a

93 complete summary of reads, misassembly can result in the contigs having an incorrect structure,

94 and for parts of the genome where contigs could not be assembled, no information is available.

95 In addition, reference-guided assembly of viral genomes can lead to biased loss of information

96 which can then skew epidemiological and evolutionary conclusions (Wymant et al., 2018).

97 Variant analysis and genome quality assessment to detect variants and changes occurring across

98 the genome of a virus is also a key step in viral genome analysis as viruses (especially RNA

99 viruses) are known to have high mutation rates (Duffy, 2018). Variant analysis is important for

100 detecting outbreak origins and for phylogenetic/phylogeographic studies and best practices for

101 variant identification in microbial genomes have been proposed in literature and adopted to a

102 large extent (Van der Auwera et al., 2013).

103 A number of pipelines that have been developed for downstream analysis of viral genomes

104 require high performance computing (HPC) clusters and/or cloud-based systems e.g. the V-pipe

105 authors recommend running V-pipe on clusters because for most applications, running V-pipe on

106 a local machine may not be efficient (https://github.com/cbg-ethz/V-pipe/wiki/advanced) and

107 some of these pipelines are only web-based such as VirAmp (Wan et al., 2015) and VirusTAP

108 (Yamashita, 2016). Also, some pipelines have many dependencies to be installed especially if the

109 analysis requires multiple tasks to be performed. In low-and-middle income countries (LMICs)

110 where most scientists do not have access to HPC clusters or cloud-based systems and where

111 internet connection is too unstable to regularly make use of web-based platforms for analysis,

112 this can be a daunting task. 
113 The challenges listed above motivated the development of VGEA (Viral Genomes Easily

114 Analyzed, available online at https://github.com/pauloluniyi/VGEA). VGEA makes use of

115 existing bioinformatics pipeline/tools to carry out various viral genome analysis tasks and is built

116 on an advanced workflow management system, Snakemake (Köster \& Rahmann, 2012).

117 MATERIALS AND METHODS

\section{Datasets}

119 We successfully tested and validated VGEA with SARS-CoV-2 $(n=20)$ and Lassa Virus $(n=$

120 20) datasets sequenced on the illumina MiSeq and illumina FGx sequencing machines in our

121 laboratory at the African Centre of Excellence for Genomics of Infectious Diseases (ACEGID),

122 Redeemer's University, Ede, Nigeria. Briefly, samples were inactivated in buffer AVL and viral

123 RNA was extracted according to the QiAmp viral RNA mini kit (Qiagen) manufacturer's

124 instructions. Extracted RNA was treated with Turbo DNase to remove contaminating DNA,

125 followed by cDNA synthesis with random hexamers. Sequencing libraries were prepared using

126 the Nextera XT kit (Illumina) as previously described_(Matranga et al., 2016) and sequenced on

127 the Illumina Miseq platform with 101 base pair paired-end reads. We also tested and validated

128 VGEA with HIV-1 datasets sequenced on the illumina HiSeq 2500 obtained from NCBI

129 Sequence Read Archive (SRA). We made use of 60 test datasets [Lassa Virus (20), SARS-CoV-

$1302(20)$ and HIV-1 (20)] for the validation of the VGEA pipeline. All our test datasets are

131 available on figshare (https://doi.org/10.6084/m9.figshare.13009997). 


\section{Implementation}

135 The installation of VGEA requires the pipeline to be downloaded onto a personal computer and

136 creation of a conda environment to set up all dependencies. Complete installation steps are in the

137 github README file:

138 https:/github.com/pauloluniyi/VGEA/blob/master/README.md

139 The analysis of VGEA is broken down into a set of 'rules' that links the output file of an analysis

140 into the input of the next task in the general workflow (Figure 1). The dependencies are fastp for

141 read trimming and read-level quality control, BWA for mapping sequencing reads to the human

142 reference genome, SAMtools for extracting unmapped reads and also for splitting bam files into

143 fastq files, IVA for de novo assembly to generate contigs, shiver to pre-process reads for quality

144 and contamination, then map to a reference tailored to the sample using corrected contigs

145 supplemented with the user's choice of existing reference sequences, SeqKit for cleaning shiver

146 assembly for QUAST, QUAST to evaluate/assess the quality of genome assemblies and

147 MultiQC for aggregation of the results from fastp, BWA and QUAST

148

149 All of these tools can be installed using a bioconda channel (Grüning et al., 2018). The input

150 files for VGEA are paired-end fastq files. VGEA allows full customization of the pipeline, so

151 users can modify the parameters used in running their samples. It is possible to modify every

152 step of the workflow to suit the samples being processed. Users can also add more steps to the

153 pipeline as they see fit. The pipeline runs on Linux/Unix and Mac. No prior programming is

154 required however to run the pipeline and once the user supplies the input, the whole workflow

155 can run automatically from beginning to end.

156

Peer) reviewing PDF | (2020:09:53134:3:0:NEW 8 Jul 2021) 


\section{RESULTS}

158

159 VGEA carries out read trimming and quality control tasks on input FASTQ data using fastp

160 (Figure 2). This increases the quality of data used for subsequent steps of the pipeline.

161 VGEA then maps reads to the human reference genome in order to remove human contaminants,

162 the pipeline carries out this step using BWA. Genome assembly and consensus sequence

163 generation is carried out, together with the generation of summary minority-variant information

164 (base frequencies at each position) and detailed minority-variant information (all reads aligned to

165 their correct position in the genome). VGEA carries out assembly using IVA and generates

166 consensus sequences using shiver. Previous study by the shiver developers has shown the

167 systematic superiority of mapping to shiver's constructed reference compared with mapping the

168 same reads to the closest of 3,249 references: median values of 13 bases called differently and

169 more accurately, zero bases called differently and less accurately, and 205 bases of missing

170 sequence recovered (Wymant et al., 2018).

172 VGEA also assesses the quality of genome assemblies using QUAST. QUAST evaluates metrics

173 such as contig sizes, misassemblies and structural variations, genome representation and its

174 functional elements, variations of N50 based on aligned blocks and then presents these statistics

175 in graphical form. QUAST also makes a histogram of several metrics including the number of

176 complete genes, operons and the genome fraction (\%). Finally, VGEA compiles the results of

177 BWA, fastp and QUAST into a single MultiQC report (Figure 3).

178

179

PeerJ reviewing PDF | (2020:09:53134:3:0:NEW 8 Jul 2021) 


\section{Performance Evaluation}

181

182 VGEA makes use of Snakemake's benchmarking feature which allows the measurement of the

183 CPU usage and wall clock time of each rule in the pipeline. This allows the user to know which

184 step of the pipeline requires the least and highest amount of computational resources. Knowledge

185 of this can help the user decide on the number of threads to dedicate to each rule as VGEA also

186 makes use of Snakemake's multi-threading feature. Table 1 shows the benchmarking values for a

187 sample SARS-CoV-2 dataset analyzed using VGEA.

188

189 We compared the contigs generated by VGEA's assembly step with contigs generated using two

190 other standalone and commonly used assembly pipelines, SPAdes (Bankevich et al., 2012) and

191 Velvet (Zerbino and Birney, 2008). We compared against these two pipelines because most

192 commonly used assembly workflows like viral-ngs and VirAmp are built on them. We carried

193 out this comparison by making use of five different SARS-CoV-2 test datasets (namely CV18,

194 CV29, CV45, CV115 and CV145 datasets available on FigShare and NCBI). We compared the

195 assemblies to the SARS-CoV-2 reference genome, and N50/NG50, mis-assembly, mismatches

196 and indel scores were used to evaluate the performance of each assembly method as

197 recommended by Assemblathon 2 (Bradnam et al., 2013) (Table 2). Basic statistics were

198 calculated using QUAST. All results of our performance evaluation and comparison are provided

199 as Supplementary File 2. All analyses were run on a 64-bit personal computer with 16GB RAM

200 using four threads. SPAdes version 3.15.2 and Velvet version 1.2.10 were used for the

201 comparison purposes using the default parameters. 
203 Evaluation statistics showed that contigs generated by VGEA had the highest NG50 score for

204 four of the five datasets and the highest N50 scores across all five datasets. In all five datasets,

205 VGEA's contigs had the highest genome fraction covering greater than $95 \%$ in four.

206 Comparison of maximum RAM used by VGEA, SPAdes and Velvet showed that VGEA used

207 the least amount of RAM for the analyses of all five datasets used for comparison. SPAdes and

208 Velvet however ran faster than VGEA for all analyses.

209

210 DISCUSSION

211

212 VGEA is built on the snakemake workflow management system (Köster \& Rahmann, 2012), a

213 workflow management system that allows the effortless deployment and execution of complex

214 distributed computational workflows in any UNIX-based system, from local machines to high-

215 performance computing clusters. It is a user-friendly, customizable and reproducible pipeline

216 which can be deployed on a personal computer and which can run from start to finish with a

217 single command.

218

219 VGEA was designed with ease-of-use in mind and so all its dependencies can be installed in a 220 conda environment under the bioconda channel (Grüning et al., 2018) making it particularly

221 useful for scientists with little or no computational background and for scientists in LMICs who

222 don't have much access to high-performance computing clusters or cloud-computing resources.

223 VGEA capitalizes on Snakemake's multi-threading feature so that makes it possible for it to be

224 deployed on laptops with greater computing performance or a computing server to improve its 
225 speed. The pipeline was tested with paired-end short-read sequencing data produced by the

226 illumina platform (MiSeq, MiSeq FGx and HiSeq 2500).

227

228 The results generated by the major steps of the VGEA pipeline are summed up together into a

229 MultiQC report which can be easily interpreted and understood by anyone with little or no

230 knowledge of bioinformatics.

231

232

233 CONCLUSION

234 VGEA was built primarily by biologists and in a manner that is easy to be employed by users

235 without significant computational background. As new and innovative tools for viral genome

236 analysis and assembly are increasingly being developed, these can easily be incorporated into the

237 VGEA pipeline. We hope that other scientists can build upon and improve VGEA as a tool to

238 extract more qualitative and quantitative information from viral genomes.

239

240

241 ACKNOWLEDGEMENTS

242 We appreciate the continuous support of ACEGID staff and the management of Redeemer's

243 University. We especially appreciate Dr. Finlay Maguire and Dr. Gerry Tonkin-Hill for helpful

244 discussions and for making necessary changes to the pipeline. Also, thanks to Dr. Andreas Wilm

245 and Christopher Tomkins-Tinch for helpful comments and suggestions. 


\section{Abbreviations}

249 VGEA Viral Genomes Easily Assembled

250 NGS Next generation sequencing

251 RNA Ribonucleic acid

252 SARS Severe Acute Respiratory Syndrome

253 MERS Middle East Respiratory Syndrome

254 IVA Iterative Virus Assembler

255 SHIVER Sequences from HIV Easily Reconstructed

256 HPC High Performance Computing

257 Data Availability

258 The following information was supplied regarding data availability:

259

VGEA is freely available on GitHub at: https://github.com/pauloluniyi/VGEA under the GNU General Public License.

261

All primary test datasets used for the validation of the VGEA pipeline are available on figshare (https://doi.org/10.6084/m9.figshare.13009997). All SARS-CoV-2 and Lassa virus test datasets

264 have been submitted to NCBI SRA (BioProject accession numbers, PRJNA666685 and

265 PRJNA666664). All HIV-1 test datasets are available on NCBI SRA (accession numbers: ERR3953696, ERR3953853, ERR3953893, ERR3953891, ERR3953866, ERR3953846, ERR3953756, ERR3953877, ERR3953876, ERR3953750, ERR3953741, ERR3953697, ERR3953699, ERR3953706, ERR3953708, ERR3953710, ERR3953712, ERR3953716, ERR3953295, ERR3953693). 


\section{REFERENCES}

274

275

Ajogbasile FV, Oguzie JU, Oluniyi PE, Eromon PE, Uwanibe JN, Mehta SB, Siddle KJ,

Odia I, Winnicki SM, Akpede N, Akpede G, Okogbenin S, Ogbaini-Emovon E, MacInnis BL, Folarin OA, Modjarrad K, Schaffner SF, Tomori O, Ihekweazu C, Sabeti PC, Happi CT. 2020. Real-time Metagenomic Analysis of Undiagnosed Fever Cases Unveils a Yellow Fever Outbreak in Edo State, Nigeria. Scientific reports 10:3180. DOI: $10.1038 / \mathrm{s} 41598-020-59880-w$.

Altschul SF, Gish W, Miller W, Myers EW, Lipman DJ. 1990. Basic local alignment search tool. Journal of molecular biology 215:403-410. DOI: 10.1016/S0022-2836(05)80360-2.

Bankevich A, Nurk S, Antipov D, Gurevich AA, Dvorkin M, Kulikov AS, Lesin VM, Nikolenko SI, Pham S, Prjibelski AD, Pyshkin AV, Sirotkin AV, Vyahhi N, Tesler G, Alekseyev MA, Pevzner PA. 2012. SPAdes: a new genome assembly algorithm and its applications to single-cell sequencing. Journal of computational biology: a journal of computational molecular cell biology 19:455-477. DOI: 10.1089/cmb.2012.0021.

Bean AGD, Baker ML, Stewart CR, Cowled C, Deffrasnes C, Wang L-F, Lowenthal JW. 
292

293

294

295

296

297

298

299

300

301

302

303

304

305

306

307

308

309

310

311

312 Chen N, Zhou M, Dong X, Qu J, Gong F, Han Y, Qiu Y, Wang J, Liu Y, Wei Y, Xia J 'an,

2013. Studying immunity to zoonotic diseases in the natural host - keeping it real. Nature reviews. Immunology 13:851-861. DOI: 10.1038/nri3551.

Bolger AM, Lohse M, Usadel B. 2014. Trimmomatic: a flexible trimmer for Illumina sequence data. Bioinformatics 30:2114-2120. DOI: 10.1093/bioinformatics/btu170.

Brister JR, Ako-Adjei D, Bao Y, Blinkova O. 2015. NCBI viral genomes resource. Nucleic acids research 43:D571-7. DOI: 10.1093/nar/gku1207.

Cantalupo PG, Calgua B, Zhao G, Hundesa A, Wier AD, Katz JP, Grabe M, Hendrix RW, Girones R, Wang D, Pipas JM. 2011. Raw sewage harbors diverse viral populations. mBio 2. DOI: 10.1128/mBio.00180-11.

Chan PKS. 2002. Outbreak of avian influenza A(H5N1) virus infection in Hong Kong in 1997. Clinical infectious diseases: an official publication of the Infectious Diseases Society of America 34 Suppl 2:S58-64. DOI: 10.1086/338820.

Chen S, Zhou Y, Chen Y, Gu J. 2018. fastp: an ultra-fast all-in-one FASTQ preprocessor. Bioinformatics 34:i884-i890. DOI: 10.1093/bioinformatics/bty560.

PeerJ reviewing PDF | (2020:09:53134:3:0:NEW 8 Jul 2021) 
313

314

315

316

317

318

319
Yu T, Zhang X, Zhang L. 2020. Epidemiological and clinical characteristics of 99 cases of 2019 novel coronavirus pneumonia in Wuhan, China: a descriptive study. The Lancet 395:507-513. DOI: 10.1016/S0140-6736(20)30211-7.

Cock PJA, Antao T, Chang JT, Chapman BA, Cox CJ, Dalke A, Friedberg I, Hamelryck T, Kauff F, Wilczynski B, de Hoon MJL. 2009. Biopython: freely available Python tools for computational molecular biology and bioinformatics. Bioinformatics 25:1422-1423. DOI: 10.1093/bioinformatics/btp163.

Duffy S. 2018. Why are RNA virus mutation rates so damn high?. PLoS biology, 16(8), e3000003. https://doi.org/10.1371/journal.pbio.3000003.

Ewels P, Magnusson M, Lundin S, Käller M. 2016. MultiQC: summarize analysis results for multiple tools and samples in a single report. Bioinformatics, 32(19):3047-3048. DOI:10.1093/bioinformatics/btw354.

\section{Folarin OA, Ehichioya D, Schaffner SF, Winnicki SM, Wohl S, Eromon P, West KL,} Gladden-Young A, Oyejide NE, Matranga CB, Deme AB, James A, Tomkins-Tinch C, Onyewurunwa K, Ladner JT, Palacios G, Nosamiefan I, Andersen KG, Omilabu S, Park DJ, Yozwiak NL, Nasidi A, Garry RF, Tomori O, Sabeti PC, Happi CT. 2016. Ebola Virus Epidemiology and Evolution in Nigeria. The Journal of infectious diseases 214:S102-S109. DOI: 10.1093/infdis/jiw190. 
336 Grubaugh ND, Ladner JT, Kraemer MUG, Dudas G, Tan AL, Gangavarapu K, Wiley MR, White S, Thézé J, Magnani DM, Prieto K, Reyes D, Bingham AM, Paul LM, Robles-Sikisaka R, Oliveira G, Pronty D, Barcellona CM, Metsky HC, Baniecki ML, Barnes KG, Chak B, Freije CA, Gladden-Young A, Gnirke A, Luo C, MacInnis B, Matranga CB, Park DJ, Qu J, Schaffner SF, Tomkins-Tinch C, West

Grüning, B., Dale, R., Sjödin, A., Chapman, B. A., Rowe, J., Tomkins-Tinch, C. H., comprehensive software distribution for the life sciences. Nature methods, 15(7), 475-476. https://doi.org/10.1038/s41592-018-0046-7.

Gurevich, A., Saveliev, V., Vyahhi, N., \& Tesler, G. 2013. QUAST: quality assessment tool for genome assemblies. Bioinformatics (Oxford, England), 29(8), 1072-1075. https://doi.org/10.1093/bioinformatics/btt086. 
Holshue ML, DeBolt C, Lindquist S, Lofy KH, Wiesman J, Bruce H, Spitters C, Ericson K, Wilkerson S, Tural A, Diaz G, Cohn A, Fox L, Patel A, Gerber SI, Kim L, Tong S, Lu X, Lindstrom S, Pallansch MA, Weldon WC, Biggs HM, Uyeki TM, Pillai SK, Washington State 2019-nCoV Case Investigation Team. 2020. First Case of 2019 Novel Coronavirus in the United States. The New England journal of medicine. DOI: 10.1056/NEJMoa2001191.

365

366

Hunt M, Gall A, Ong SH, Brener J, Ferns B, Goulder P, Nastouli E, Keane JA, Kellam P, Otto TD. 2015. IVA: accurate de novo assembly of RNA virus genomes. Bioinformatics 31:2374-2376. DOI: 10.1093/bioinformatics/btv120. sequence alignment based on fast Fourier transform. Nucleic acids research 30:30593066. DOI: $10.1093 / \mathrm{nar} / \mathrm{gkf436.}$ statistics. Bioinformatics 33:2759-2761. DOI: 10.1093/bioinformatics/btx304.

Köster J, Rahmann S. 2012. Snakemake--a scalable bioinformatics workflow engine. 
380 Langmead B. 2010. Aligning short sequencing reads with Bowtie. Current protocols in bioinformatics / editoral board, Andreas D. Baxevanis... [et al.] Chapter 11:Unit 11.7. DOI: 10.1002/0471250953.bi1107s32.

383

Li H, Durbin R. 2009. Fast and accurate short read alignment with Burrows-Wheeler transform. Bioinformatics 25:1754-1760. DOI: 10.1093/bioinformatics/btp324.

386

\section{Li H, Handsaker B, Wysoker A, Fennell T, Ruan J, Homer N, Marth G, Abecasis G,} Durbin R, 1000 Genome Project Data Processing Subgroup. 2009. The Sequence Alignment/Map format and SAMtools. Bioinformatics 25:2078-2079. DOI: 10.1093/bioinformatics/btp352.

391

Marçais G, Delcher AL, Phillippy AM, Coston R, Salzberg SL, Zimin A. 2018. MUMmer4:

Matranga, C. B., Gladden-Young, A., Qu, J., Winnicki, S., Nosamiefan, D., Levin, J. A fast and versatile genome alignment system. PLoS computational biology 14:e1005944. DOI: 10.1371/journal.pcbi.1005944. Z., \& Sabeti, P. C. 2016. Unbiased Deep Sequencing of RNA Viruses from 400 

Valim C, Degrave W, Bozza PT, Gnirke A, Andersen KG, Isern S, Michael SF,

414 Mokili JL, Rohwer F, Dutilh BE. 2012. Metagenomics and future perspectives in virus virus evolution and spread in the Americas. Nature 546:411-415. DOI:

417 Nakamura, Y., Yasuike, M., Nishiki, I., Iwasaki, Y., Fujiwara, A., Kawato, Y., Nakai, 10.1038/nature22402. T., Nagai, S., Kobayashi, T., Gojobori, T., \& Ototake, M. 2016. V-GAP: Viral genome assembly pipeline. Gene, 576(2 Pt 1), 676-680. 
JT, Kugelman JR, Matranga CB, Winnicki SM, Qu J, Gire SK, Gladden-Young A,

424

425

426

427

428

429

430

431

432

433

434

435

436

437

438

439

440

441

442

443

444

445

Jalloh S, Nosamiefan D, Yozwiak NL, Moses LM, Jiang P-P, Lin AE, Schaffner SF,

Bird B, Towner J, Mamoh M, Gbakie M, Kanneh L, Kargbo D, Massally JLB,

Kamara FK, Konuwa E, Sellu J, Jalloh AA, Mustapha I, Foday M, Yillah M,

Erickson BR, Sealy T, Blau D, Paddock C, Brault A, Amman B, Basile J, Bearden S,

Belser J, Bergeron E, Campbell S, Chakrabarti A, Dodd K, Flint M, Gibbons A,

Goodman C, Klena J, McMullan L, Morgan L, Russell B, Salzer J, Sanchez A,

Wang D, Jungreis I, Tomkins-Tinch C, Kislyuk A, Lin MF, Chapman S, MacInnis

B, Matthews A, Bochicchio J, Hensley LE, Kuhn JH, Nusbaum C, Schieffelin JS,

Birren BW, Forget M, Nichol ST, Palacios GF, Ndiaye D, Happi C, Gevao SM,

Vandi MA, Kargbo B, Holmes EC, Bedford T, Gnirke A, Ströher U, Rambaut A,

Garry RF, Sabeti PC. 2015. Ebola Virus Epidemiology, Transmission, and Evolution

during Seven Months in Sierra Leone. Cell 161:1516-1526. DOI:

10.1016/j.cell.2015.06.007.

Pickett BE, Sadat EL, Zhang Y, Noronha JM, Squires RB, Hunt V, Liu M, Kumar S, Zaremba S, Gu Z, Zhou L, Larson CN, Dietrich J, Klem EB, Scheuermann RH. 2012. ViPR: an open bioinformatics database and analysis resource for virology research. Nucleic acids research 40:D593-8. DOI: 10.1093/nar/gkr859.

Posada-Céspedes, S., Seifert, D., Topolsky, I., Jablonski, K. P., Metzner, K. J., \& Beerenwinkel, N. 2021. V-pipe: a computational pipeline for assessing viral genetic diversity from high-throughput data. Bioinformatics (Oxford, England),

Peer] reviewing PDF | (2020:09:53134:3:0:NEW 8 Jul 2021) 
446

447 448 449

453

454 btab015. Advance online publication. https://doi.org/10.1093/bioinformatics/btab015

Reyes A, Haynes M, Hanson N, Angly FE, Heath AC, Rohwer F, Gordon JI. 2010. Viruses in the faecal microbiota of monozygotic twins and their mothers. Nature 466:334-338. DOI: $10.1038 /$ nature09199.

Sharma D, Priyadarshini P, Vrati S. 2015. Unraveling the web of viroinformatics: computational tools and databases in virus research. Journal of virology 89:1489-1501. DOI: 10.1128/JVI.02027-14.

Shen W, Le S, Li Y, Hu F. 2016. SeqKit: A Cross-Platform and Ultrafast Toolkit for FASTA/Q File Manipulation. PLoS One 11(10):e0163962. DOI:10.1371/journal.pone.0163962.

Siddle KJ, Eromon P, Barnes KG, Mehta S, Oguzie JU, Odia I, Schaffner SF, Winnicki SM, Shah RR, Qu J, Wohl S, Brehio P, Iruolagbe C, Aiyepada J, Uyigue E, Akhilomen P, Okonofua G, Ye S, Kayode T, Ajogbasile F, Uwanibe J, Gaye A, Momoh M, Chak B, Kotliar D, Carter A, Gladden-Young A, Freije CA, Omoregie O, Osiemi B, Muoebonam EB, Airende M, Enigbe R, Ebo B, Nosamiefan I, Oluniyi P, Nekoui M, Ogbaini-Emovon E, Garry RF, Andersen KG, Park DJ, Yozwiak NL, Akpede G, Ihekweazu C, Tomori O, Okogbenin S, Folarin OA, Okokhere PO, 
468

469

470

471

472

473

474

475

476

477

478

479

480

481

482

483

484

485

486

487

488

489

MacInnis BL, Sabeti PC, Happi CT. 2018. Genomic Analysis of Lassa Virus during an Increase in Cases in Nigeria in 2018. The New England journal of medicine 379:17451753. DOI: $10.1056 /$ NEJMoa1804498.

Sohrabi C, Alsafi Z, O’Neill N, Khan M, Kerwan A, Al-Jabir A, Iosifidis C, Agha R. 2020. World Health Organization declares Global Emergency: A review of the 2019 Novel Coronavirus (COVID-19). International journal of surgery. DOI:

10.1016/j.ijsu.2020.02.034.

Tang P, Chiu C. 2010. Metagenomics for the discovery of novel human viruses. Future microbiology 5:177-189. DOI: 10.2217/fmb.09.120.

Van der Auwera GA, Carneiro MO, Hartl C, Poplin R, Del Angel G, Levy-Moonshine A, Jordan T, Shakir K, Roazen D, Thibault J, Banks E, Garimella KV, Altshuler D, Gabriel S, DePristo MA. 2013. From FastQ data to high confidence variant calls: the Genome Analysis Toolkit best practices pipeline. Curr Protoc Bioinformatics. 43(1110):11.10.1-11.10.33. doi: 10.1002/0471250953.bi1110s43.

Wan Y, Renner DW, Albert I, Szpara ML. 2015. VirAmp: a galaxy-based viral genome assembly pipeline. Gigascience. 4:19. doi:10.1186/s13742-015-0060-y.

Wymant C, Blanquart F, Golubchik T, Gall A, Bakker M, Bezemer D, Croucher NJ, Hall 
497 Yamashita, A., Sekizuka, T., \& Kuroda, M. 2016. VirusTAP: Viral Genome-Targeted

498 Assembly Pipeline. Frontiers in microbiology, 7, 32

499

https://doi.org/10.3389/fmicb.2016.00032.

500

501

502

503 


\section{Figure 1}

\section{A schematic workflow of VGEA}

User-supplied paired-end fastq files are pre-processed and trimmed using FASTP followed by mapping to the human reference genome with BWA. Following mapping, a BAM file containing unaligned/unmapped reads is extracted using SAMTOOLS. This BAM file is then split into fastq files of forward and reverse reads also with SAMTOOLS after which de novo assembly is carried out using IVA. Following de novo assembly, SHIVER is used to map the reads and generate consensus sequences, and detailed minority variant information (full explanation of the shiver method is in supplementary file 1). SEQKIT is used to clean the SHIVER output for QUAST after which genome evaluation and assessment is carried out using QUAST. MULTIQC is then used for aggregation of results from BWA, FASTP and QUAST. 


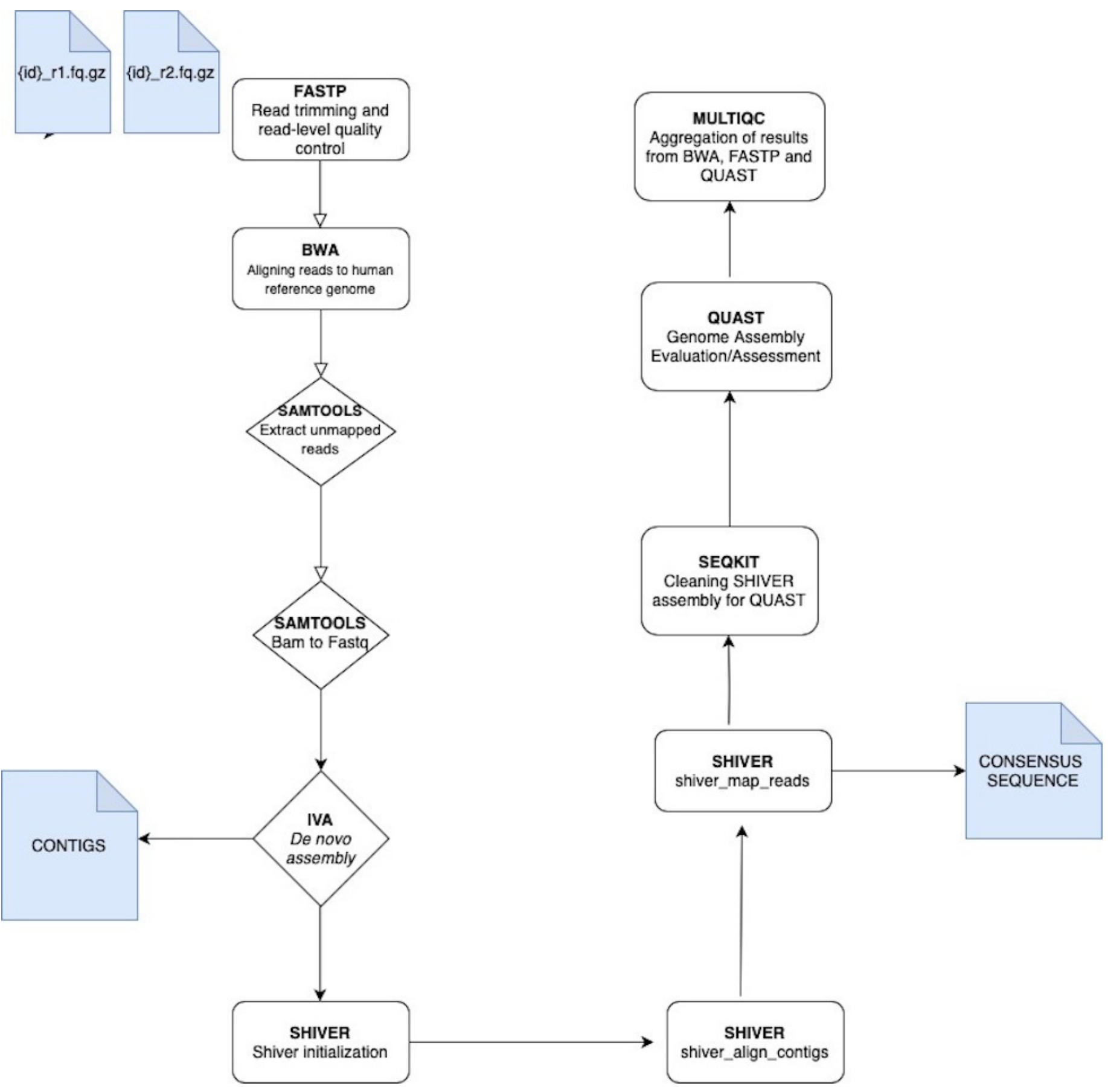


Figure 2

fastp pre-processing report for a SARS-CoV-2 test dataset analyzed using VGEA

\section{Before filtering}

Before filtering: read1: quality

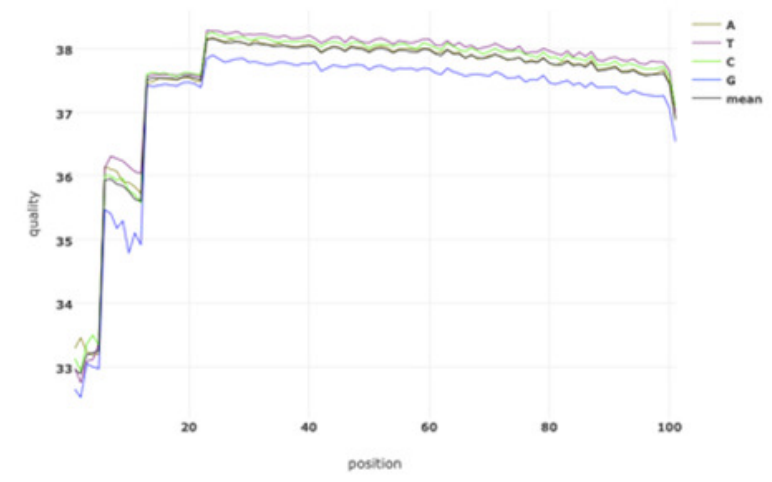

Before filtering: read2: quality

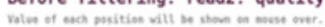

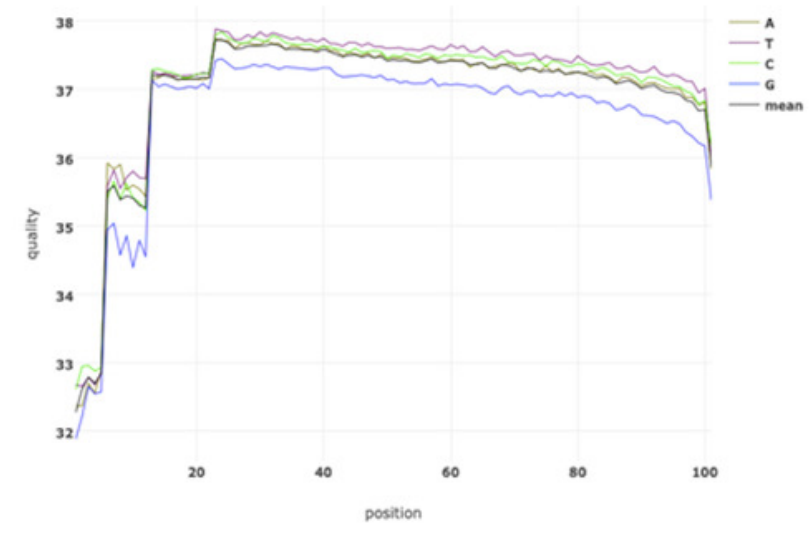

\section{After filtering}

After filtering: read1: quality

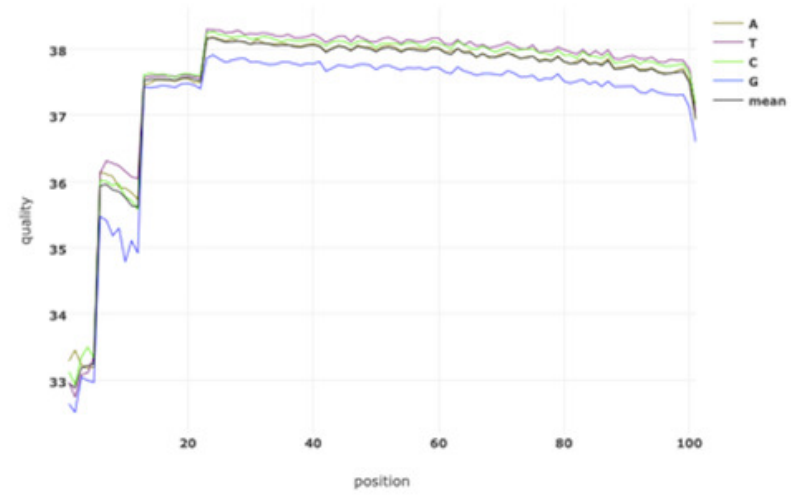

After filtering: read2: quality

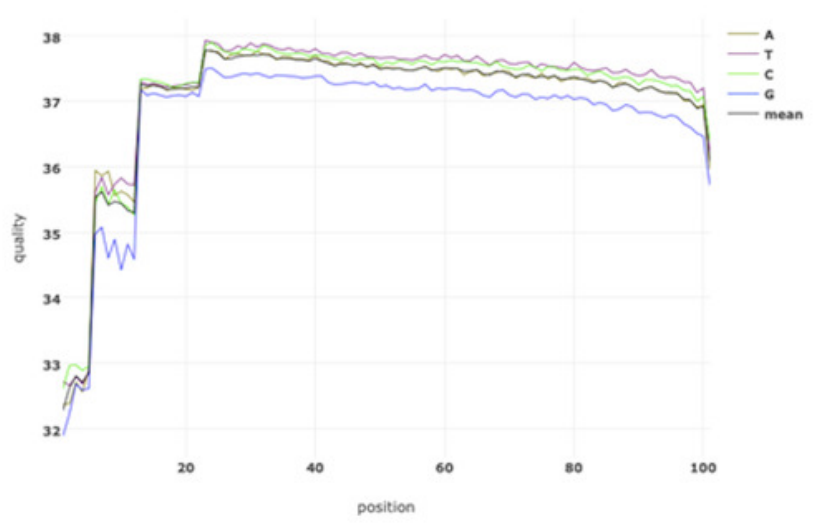


Figure 3

\section{MultiQC report of five SARS-CoV-2 datasets analyzed using VGEA}

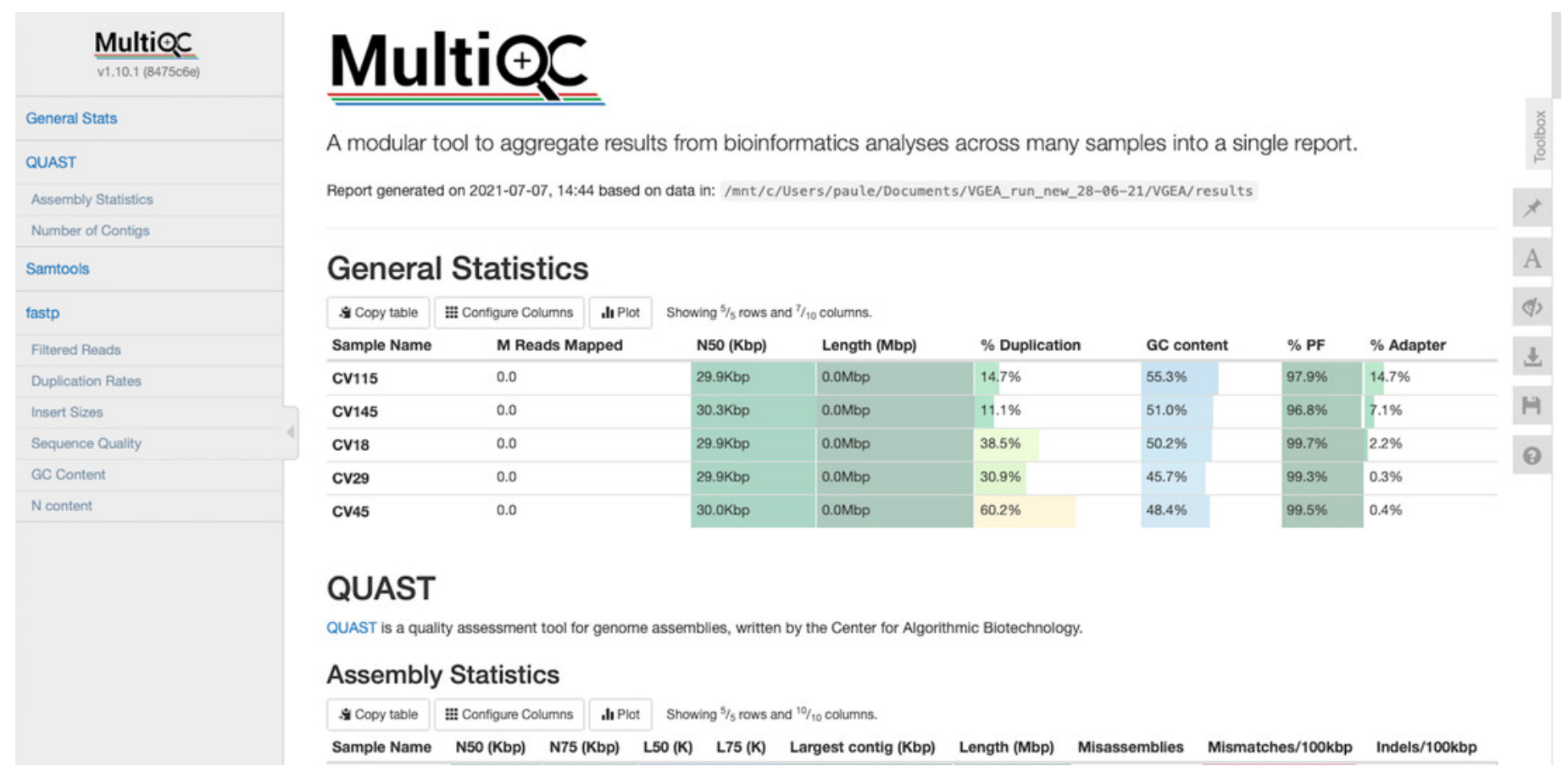




\section{Table $\mathbf{1}$ (on next page)}

Benchmarking values (time and CPU usage) for a SARS-CoV-2 dataset analyzed using VGEA 
1

\begin{tabular}{|l|l|l|}
\hline VGEA rule name & Time (h:m:s) & Maximum RAM used (MB) \\
\hline human_reference_index & $1: 01: 53$ & 4688.56 \\
\hline fastp & $0: 00: 14$ & 581.91 \\
\hline bwa_human & $0: 08: 52$ & 5960.95 \\
\hline samtools_extract & $0: 02: 40$ & 16.21 \\
\hline bamtofastq & $0: 01: 39$ & 6.61 \\
\hline *iva & $8: 19: 11$ & 238.57 \\
\hline shiver_init & $0: 00: 53$ & 64.97 \\
\hline shiver_align_contigs & $0: 04: 37$ & 2509.64 \\
\hline shiver_map_reads & $0: 31: 51$ & 567.27 \\
\hline shiver_tidy & $0: 00: 00$ & 1.06 \\
\hline quast & $0: 00: 33$ & 72.51 \\
\hline
\end{tabular}

2 *IVA was run using one CPU core and two threads so if allowed more computational resources, the assembly time 3 will be even shorter. 
Table 2 (on next page)

Performance comparison using different assembly pipelines 


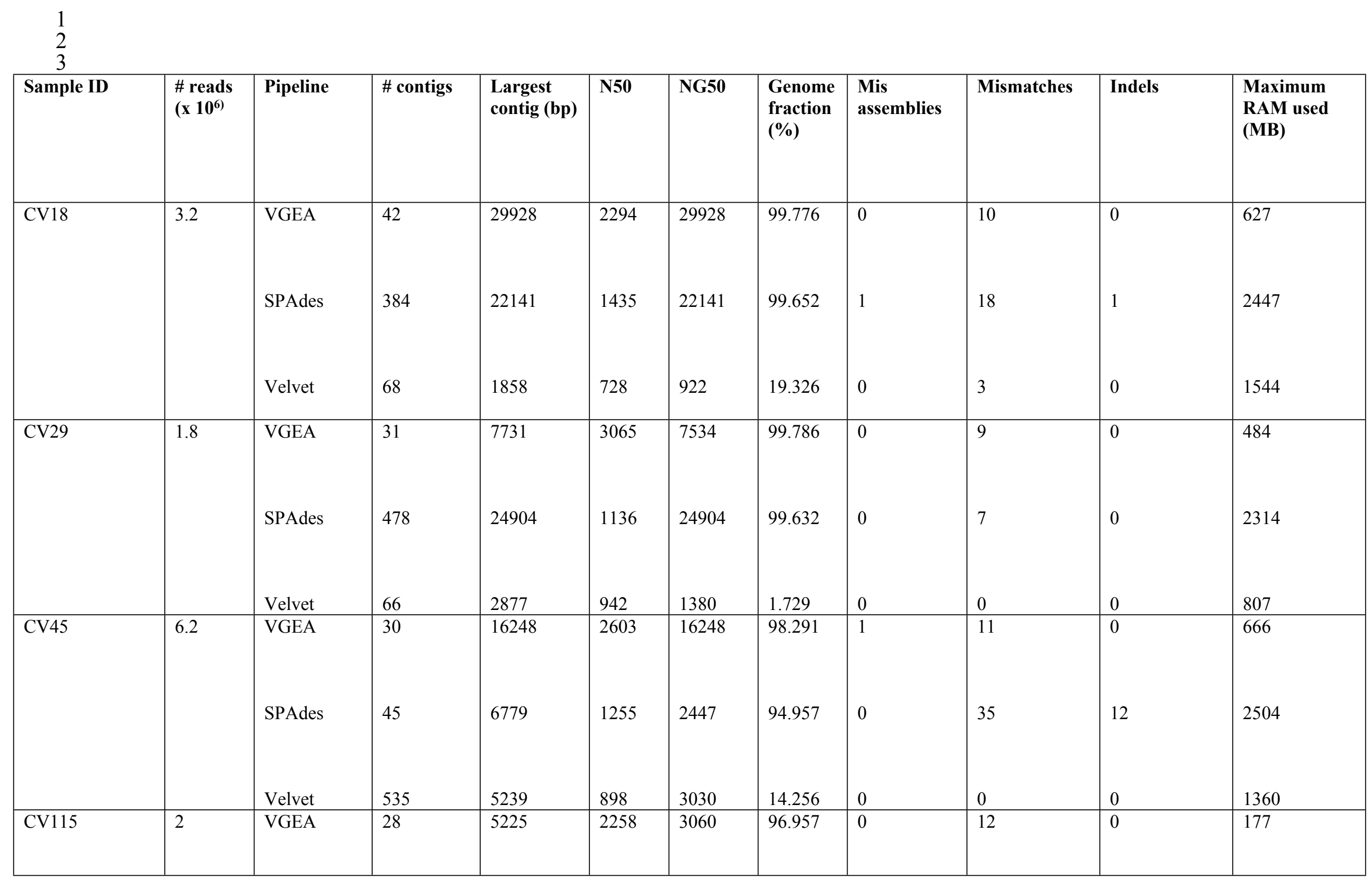




\begin{tabular}{|c|c|c|c|c|c|c|c|c|c|c|c|}
\hline & & *SPAdes & 49 & 1942 & 1068 & 1828 & - & - & - & - & 1735 \\
\hline & & Velvet & 41 & 2847 & 819 & 931 & 68.134 & 0 & 9 & 0 & 511 \\
\hline \multirow[t]{3}{*}{ CV145 } & \multirow[t]{3}{*}{4.4} & VGEA & 28 & 6807 & 2049 & 4214 & 73.093 & 0 & 14 & 0 & 635 \\
\hline & & SPAdes & 188 & 3216 & 1190 & 2477 & 5.073 & 2 & 13 & 0 & 2547 \\
\hline & & Velvet & 178 & 1798 & 682 & 1107 & 3.578 & 0 & 0 & 0 & 1459 \\
\hline
\end{tabular}

$4 \quad *$ QUAST gave no genome fraction value for this sample 